\title{
Probability Distribution of Polymethylene Chains ${ }^{\dagger}$
}

\author{
Linxi ZHANG, ${ }^{*}$ Jianmin Xu, and Agen XIA \\ Department of Physics, Hangzhou University, Hangzhou, \\ People's Republic of China
}

(Received June 11, 1990)

\begin{abstract}
The probability distribution $P(S)$ of the radius of gyration of polymer chains was studied with a rotational-isomeric-state model. A Monte Carlo investigation provided reliable numerical data for $P(S)$, which was then compared with results from relatively analytic study. The method was to maximize entropy functional $P(S)$ subject to assigned $\left\langle S^{2}\right\rangle$ and $\left\langle S^{4}\right\rangle$. The results for $P(S)$ were in good agreement with Monte Carlo results for large $S$.
\end{abstract}

KEY WORDS Probability Distribution / Maximum-Entropy Method /

Rotational-Isomeric-State Model / Monte Carlo Simulation /

The distribution function $P(S)$ is one of the most interesting conformational properties of a chain molecule. Fixman ${ }^{1}$ and Forsman and Hughes $^{2}$ first investigated the distribution function $P(S)$. However, Fixman's results involve some error, and complete numerical results have been obtained by Koyama, ${ }^{3}$ by Hoffman and Forsman, ${ }^{4}$ and by Fujita and Norisuye. ${ }^{5}$ But the details of the distribution function $P(S)$ of an unperturbed polymer chain are not yet fully explored while the distribution function $P(R)$ of a very small number of bonds of polymethylene chains ${ }^{6}$ was given accurately. In another paper, ${ }^{7}$ we studied the function $P(S)$ of the random-flight chain by Monte Carlo method. However, the distribution function $P(S)$ of polymethylene chain has not been explored yet. In this paper, we investigate the function $P(S)$ of polymethylene chain using the rotational-isomeric-state model and Monte Carlo method.

We know that the most direct and detailed results concerning $P(S)$ were obtained by the Monte Carlo method. In Section MONTE
CARlo CAlCulation, a Monte Carlo study of the rotational Isomeric chain on a tetrahedral lattice is described. Although the tetrahedral angle $\left(109.5^{\circ}\right)$ is not quite the angle favored for the polymethylene chain $\left(112^{\circ}\right)$, the values are close enough to provide useful insight and to make approximate analytic investigations. In Section MAXIUM ENTROPY CALCULATION, an alternative to the Hermite relationship between $P(S)$ and its moments is investigated. This alternative is based on a maximum-entropy formulation of the moment problem. The maximum-entropy results agree reasonably well with those of the Monte Carlo method for large $S$. Ratios of moments of the radii of gyration of maximumentropy method agree well with those calculated by the Monte Carlo method and the deviation is less than 0.053 .

We conclude from this, first, that Monte Carlo method is useful for calculating the distribution function. Secondly, we conclude that the maximum-entropy method is accurate for $P(S)$ for large $S$.

${ }^{+}$Project Supported by the Science Fund of the Scientific and Technical Commission of Zhejiang Province.

* To whom all correspondence should be addressed. 


\section{MONTE CARLO CALCULATION}

\section{Chain Model}

The rotational-isomeric-state (RIS) description of the unperturbed dimensions of polymer chains $^{8}$ is widely accepted as satisfactory for interpreting mean-squared end-to-end distance $\left(\left\langle\mathrm{R}^{2}\right\rangle_{0}\right)$ and temperature coefficients $\left(\mathrm{d} \ln \left\langle\mathrm{R}^{2}\right\rangle_{0} / \mathrm{d} T\right)$ of the polymethylene chains. In this model, the $\mathrm{C}-\mathrm{C}$ bond length $(l=1.53 \AA)$ and CCC bond-angle supplement $\left(\theta=68^{\circ}\right)$ are both assumed to be fixed. The internal rotational angle $\phi$ about each skeletal bond measured from the trans position is assumed to take three discrete values, trans $\left(\phi_{\mathrm{t}}=0^{\circ}\right)$, gauche $\left(\phi_{\mathrm{g}^{+}}=120^{\circ}\right)$ and gauche prime $\left(\phi_{\mathrm{g}^{-}}=\right.$ $\left.-120^{\circ}\right)$. The energy of a gauche conformation following either a gauche conformation with the same rotational angle or the trans conformation is assumed to exceed that of the trans conformation $E_{\sigma}$, whereas the energy of a gauche conformation following a gauche conformation with the opposite rotational angle is assumed to exceed that of the trans conformation by $E_{\mathrm{G}}$. In our calculation, the polymethylene (PM) chains are generated by using the tetrahedral lattice model, which was first described by Smith et al., ${ }^{9-11}$ and described in detail in our another paper. ${ }^{12}$ The tetrahedral lattice model gives a reasonable approximation to the rotational state of the carbon backbone bond.

The statistical-weight matrix of the PM chain is given by

$$
U=\left(\begin{array}{ccc}
1 & \sigma & \sigma \\
1 & \sigma & \sigma \omega \\
1 & \sigma \omega & \sigma
\end{array}\right)
$$

where

$$
\sigma=\exp \left(-E_{\sigma} / k T\right), \quad \omega=\exp \left(-\left(E_{\mathrm{G}}-E_{\sigma}\right) / k T\right)
$$

comprise the elements of a matrix $U$. That is, the element $U_{\mathrm{rs}, \mathrm{i}}$ of this matrix is the appropriate factor for bond $i$ in state $s$ when bond $i-1$ has been assigned previously to state $r$. The indices $r$ and $s$ index the rotational states in the order $t, g^{+}$, and $g^{-}$. Thus, the probabilities of the conformation of bond $i$ from the conformation of bond $i-1$ and eq 1 are

$$
\begin{aligned}
& P_{t t}=1 /(1+2 \sigma) \\
& P_{t g^{ \pm}}=\sigma /(1+2 \sigma) \quad(\text { for } i \geqslant 4), \\
& P_{g^{ \pm} t}=1 /(1+\sigma+\sigma \omega) \\
& P_{g^{ \pm} g^{ \pm}}=\sigma /(1+\sigma+\sigma \omega) \\
& P_{g^{ \pm} g^{\mp}}=\sigma \omega /(1+\sigma+\sigma \omega) \quad(\text { for } i \geqslant 4)
\end{aligned}
$$

For $i=3$

$$
P_{t}=P_{g^{ \pm}}=1 / 3
$$

\section{Calculation Method}

If $C_{\mathrm{N}}$ is the number of samples and $f_{\mathrm{N}}(S) \Delta S$ is the number of walks whose radii of gyration lie between $S$ and $S+\Delta S$, we have

$$
P(S+\Delta S / 2)=C_{\mathrm{N}}^{-1} f_{\mathrm{N}}(S)
$$

$\Delta S$ is equal to $0.025\left\langle\mathrm{~S}^{2}\right\rangle^{1 / 2}$ in our calculation. For convenience, we change the variable from $S$ to $\eta=\left\langle\mathrm{S}^{2}\right\rangle^{-1 / 2} \cdot S$ and plot

$$
P(\eta)=\left\langle\mathrm{S}^{2}\right\rangle^{1 / 2} \cdot P(S)
$$

against $\eta$, instead of $P(S)$ vs. $S$. We express the ratio $\left\langle S^{2 p}\right\rangle /\left\langle S^{2}\right\rangle^{p}$ in teams of a new variable defined by

$$
d_{\mathrm{p}}=\int_{0}^{\infty} P(\eta) \cdot \eta^{2 \mathfrak{p}} d \eta
$$

In fact, $d_{\mathrm{p}}$ is equal to the ratio $\left\langle\mathrm{S}^{2 \mathrm{p}}\right\rangle /\left\langle\mathrm{S}^{2}\right\rangle^{\mathrm{p}}$.

\section{Results}

In this paper, calculations were carried out using $\sigma=0.543$ and $\omega=0.088$, which correspond, respectively, to $E_{\sigma}=500 \mathrm{cal} \mathrm{mol}^{-1}$ and $E_{\mathrm{G}}=2500 \mathrm{cal} \mathrm{mol}^{-1}$ at $140^{\circ} \mathrm{C}$. We used 200000 samples and calculated the function for chains of length from 50 to 500 bonds. Results for $P(\eta)$ are represented in Figure 1 for $N=100$, 200 , and 400 . In Figure 1, function $P(\eta)$ is equal 

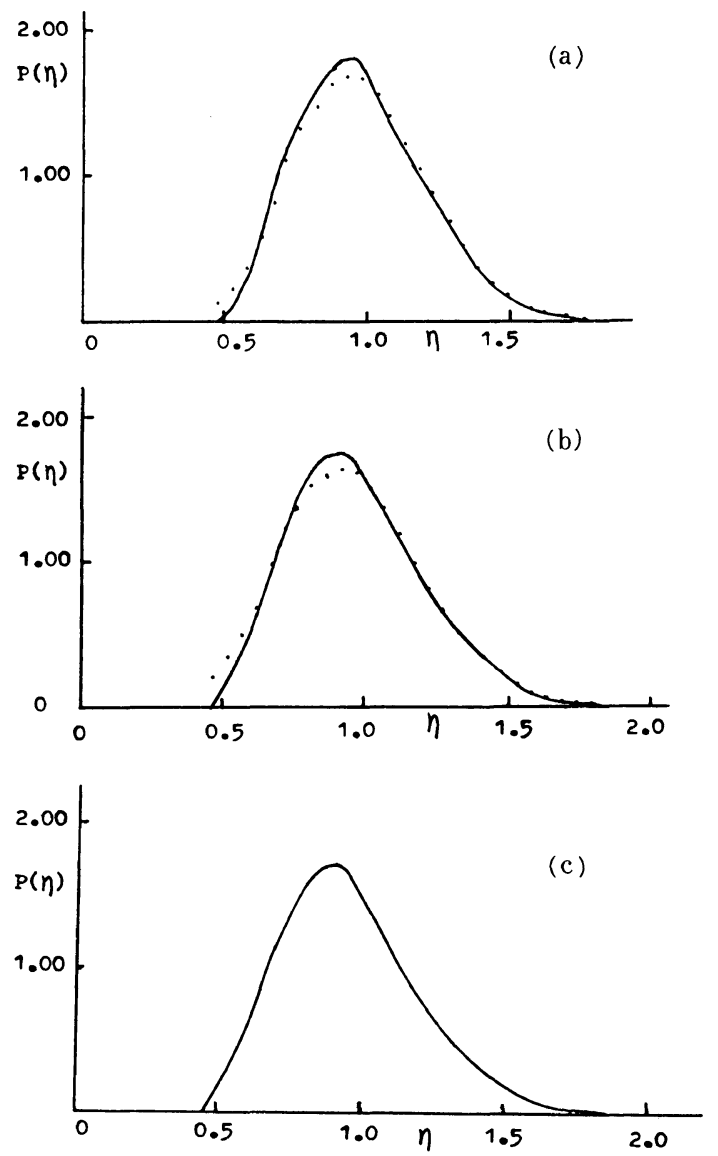

Figure 1. Probability density function $P(\eta)$ for chains of different $N$ as a function of $\eta$ for the rotational-isomeric chain on a tetrahedral lattice. The parameters in the statistical weight matrix are $\sigma=0.543$ and $\omega=0.088$. The dots are from the maximum-entropy calculation. (a) $N=100,\left\langle\mathrm{~S}^{2}\right\rangle=192.3 \AA^{2}$; (b) $N=200,\left\langle\mathrm{~S}^{2}\right\rangle=407.5 \AA^{2}$; (c) $N=400,\left\langle\mathrm{~S}^{2}\right\rangle=849.0 \AA^{2}$.

to 0 for $\eta<0.455 . P(\eta)$ for 200 bonds is greater than that for 400 bonds for $0.75<\eta<1.25$, and $P(\eta)$ for 200 bonds is less that for 400 bonds for $0.455<\eta<0.75$. The maximum of $P(\eta)$ of $\mathrm{PM}$ for 200 bonds is greater than that for 400 bonds, and less than for 100 bonds. However difference between 200 bonds and 400 bonds is small. From this, we conclude that $P(\eta)$ for $N>400$ is the same.

\section{MAXIMUM-ENTROPY CALCULATION}

One approach to statistical inference is through maximization of entropy $H$ associated with the distribution. Here,

$$
H=-\int_{0}^{L} P(S) \ln P(S) \mathrm{d} S,
$$

where $L$ is the maximum values of $S$. When $H$ is maximized subject to contraints on the average values of certain function of $S, P(S)$ becomes the exponential of a linear combination of these functions.

We assume $P(S)$ may be written as the second and fourth moments of $S$ from our calculation using Monte Carlo simulation and RIS model.

$$
\begin{array}{ll}
P(S) \sim S^{\mathrm{a}} \exp \left(-b S^{2} /\left\langle\mathrm{S}^{2}\right\rangle-c S^{4} /\left\langle\mathrm{S}^{4}\right\rangle\right) \\
& \left(S>0.455\left\langle\mathrm{~S}^{2}\right\rangle^{1 / 2}\right) \\
P(S) \sim 0 & \left(S<0.455\left\langle\mathrm{~S}^{2}\right\rangle^{1 / 2}\right)
\end{array}
$$

The moments are conveniently expressed in terms of

$$
J=\int_{X_{0}}^{X} x^{\mathrm{a}} \exp \left(-b x^{2}-c x^{4}\right) \mathrm{d} x
$$

where

$$
\begin{aligned}
x & =S /\left\langle\mathrm{S}^{2}\right\rangle^{1 / 2} \\
X & =L /\left\langle\mathrm{S}^{2}\right\rangle^{1 / 2} \\
X_{0} & =0.455 .
\end{aligned}
$$

For example,

$$
\left\langle\mathrm{S}^{4}\right\rangle /\left\langle\mathrm{S}^{2}\right\rangle^{2}=J_{\mathrm{a}+4} \cdot J_{\mathrm{a}} /\left(J_{\mathrm{a}+2}\right)^{2}
$$

and generally

$$
\left\langle\mathrm{S}^{2 \mathrm{p}}\right\rangle=J_{\mathrm{a}+2 \mathrm{p}} / J_{\mathrm{a}}
$$

The $J_{\mathrm{a}} \mathrm{s}$ are computed by numerical integration.

A numerical test of this approach was carried out for the rotational-isomeric-state model on the tetrahedral lattice. In Table I, we calculate the ratios of moments of the radii of gyration by using the maximum-entropy method and the Monte Carlo method, and find that the deviation is less than 0.053 . Function $P(\eta)$ for 
Table I. Ratios of moments of the radii of gyration $\sigma=0.543$, $\omega=0.088$

\begin{tabular}{|c|c|c|c|c|}
\hline & \multicolumn{4}{|c|}{$N=100$} \\
\hline & RIS model ${ }^{\mathrm{a}}$ & $\begin{array}{l}\text { Monte Carlo } \\
\text { calculation }\end{array}$ & $\begin{array}{l}\text { Calculated from } \\
\text { eq } 12 \text { and } 14 \\
(a=8.0, b=4.4 \\
c=0.035)\end{array}$ & Deviation \\
\hline \multirow{7}{*}{$\begin{array}{l}\left\langle\mathrm{S}^{2}\right\rangle / \mathrm{N}^{2} \\
\left\langle\mathrm{~S}^{4}\right\rangle /\left\langle\mathrm{S}^{2}\right\rangle^{2} \\
\left\langle\mathrm{~S}^{6}\right\rangle /\left\langle\mathrm{S}^{2}\right\rangle^{3} \\
\left\langle\mathrm{~S}^{8}\right\rangle /\left\langle\mathrm{S}^{2}\right\rangle^{4} \\
\left\langle\mathrm{~S}^{10}\right\rangle /\left\langle\mathrm{S}^{2}\right\rangle^{5}\end{array}$} & 0.8263 & 0.8213 & 0.8283 & 0.0085 \\
\hline & & 1.2044 & 1.2286 & 0.020 \\
\hline & & 1.7023 & 1.7511 & 0.029 \\
\hline & & 2.7482 & 2.8434 & 0.035 \\
\hline & & 4.9436 & 5.1431 & 0.040 \\
\hline & \multicolumn{4}{|c|}{$N=200$} \\
\hline & RIS model ${ }^{a}$ & $\begin{array}{l}\text { Monte Carlo } \\
\text { calculation }\end{array}$ & $\begin{array}{l}\text { Calculated from } \\
\text { eq } 12 \text { and } 14 \\
(a=6.75, b=3.8, \\
c=0.032)\end{array}$ & Deviation \\
\hline \multirow{7}{*}{$\begin{array}{l}\left\langle\mathrm{S}^{2}\right\rangle / \mathrm{N} 1^{2} \\
\left\langle\mathrm{~S}^{4}\right\rangle /\left\langle\mathrm{S}^{2}\right\rangle^{2} \\
\left\langle\mathrm{~S}^{6}\right\rangle /\left\langle\mathrm{S}^{2}\right\rangle^{3} \\
\left\langle\mathrm{~S}^{8}\right\rangle /\left\langle\mathrm{S}^{2}\right\rangle^{4} \\
\left\langle\mathrm{~S}^{10}\right\rangle /\left\langle\mathrm{S}^{2}\right\rangle^{5}\end{array}$} & 0.8809 & 0.8704 & 0.8789 & 0.0097 \\
\hline & & 1.2347 & 1.2620 & 0.022 \\
\hline & & 1.8529 & 1.8808 & 0.015 \\
\hline & & 3.2729 & 3.2394 & -0.010 \\
\hline & & 6.6350 & 6.2843 & -0.053 \\
\hline & \multicolumn{4}{|c|}{$N=400$} \\
\hline & RIS model ${ }^{\mathrm{a}}$ & $\begin{array}{l}\text { Monte Carlo } \\
\text { calculation }\end{array}$ & $\begin{array}{c}\text { Calculated from } \\
\text { eq } 12 \text { and } 14 \\
(a=5.6, b=3.3 \\
c=0.03)\end{array}$ & Deviation \\
\hline$\left\langle\mathrm{S}^{2}\right\rangle / \mathrm{N}^{2}$ & 0.9091 & 0.9067 & 0.9033 & -0.0035 \\
\hline$\left\langle\mathrm{S}^{4}\right\rangle /\left\langle\mathrm{S}^{2}\right\rangle^{2}$ & & 1.2417 & 1.2591 & 0.014 \\
\hline$\left\langle\mathrm{S}^{6}\right\rangle /\left\langle\mathrm{S}^{2}\right\rangle^{3}$ & & 1.8728 & 1.9300 & 0.031 \\
\hline$\left\langle\mathrm{S}^{8}\right\rangle /\left\langle\mathrm{S}^{2}\right\rangle^{4}$ & & 3.3756 & 3.4503 & 0.022 \\
\hline$\left\langle\mathrm{S}^{10}\right\rangle /\left\langle\mathrm{S}^{2}\right\rangle^{5}$ & & 6.8872 & 6.9716 & 0.012 \\
\hline
\end{tabular}

a Matrix multiplication calculation. $\angle \mathrm{CCC}=109.5^{\circ}$.

various $N$ are compared with the Monte Carlo results in Figure 1, where it may be seen that this maximum-entropy method is remarkably effective for representing the main features of $P(S)$ for large $S$ for all $N$.

\section{REFERENCES}

1. M. Fixman, J. Chem. Phys., 36, 306 (1962).

2. W. C. Forsman and R. E. Hughes, J. Chem. Phys.,
38, 2118 (1963); ibid., 42, 2829 (1965).

3. R. Koyama, J. Phys. Soc. Jpn., 25, 580 (1968).

4. R. B. Hoffman and W. C. Forsman, J. Chem. Phys., 50, 2316 (1969).

5. H. Fujita and T. Norisuye, J. Chem. Phys., 52, 1115 (1970).

6. M. Freire and M. Fixman, J. Chem. Phys., 69, 634 (1978).

7. Z. Linxi and X. Jiamin, Polym. J., 22, 426 (1990).

8. P. J. Flory, "Statistical Mechanics of Chain Molecules," Wiley, New York, N.Y., 1969.

9. R. P. Smith, J. Chem. Phys., 42, 1162 (1965). 


\section{Probability Distribution of Polymethylene Chains}

10. R. P. Smith and E. M. Mortensen, J. Chem. Phys., 35, 714 (1961).

11. R. P. Smith, J. Polym. Sci., 4, 869 (1966).
12. X. Jianmin, S. Xubing, and Z. Zhiping, Eur. Polym. J., 25, 601 (1989). 